\title{
Commentary
}

\section{Rising incidence of musculoskeletal discomfort in the wake of the COVID-19 crisis}

\author{
Amirhossein Memari $^{\mathrm{a}}$, Ardalan Shariat ${ }^{\mathrm{a}, *}$ and Albert Thomas Anastasio ${ }^{\mathrm{b}}$ \\ ${ }^{a}$ Sports Medicine Research Center, Neuroscience Institute, Tehran University of Medical Sciences, Tehran, Iran \\ ${ }^{\mathrm{b}}$ Department of Orthopaedic Surgery, Duke University Medical Center, Durham, USA
}

Received 13 June 2020

Accepted 25 June 2020

\begin{abstract}
The COVID-19 pandemic has led to the shutdown of much of the world's economic and social operations. Given shutdown of exercise facilities, there has been a sharp uptick in a sedentary lifestyle. As people have lost their normal daily activity patterns, it is reasonable to assume that musculoskeletal pain-related syndromes will consequently begin to increase. In addition, there has been a rise in social network, television, and online home-based workouts. In the wake of the COVID-19 pandemic, it is unclear whether previous recommendations for physical activities will remain sufficient, given cessation of normal physical activities from day-to-day life. We raise a variety of questions in dealing with the potential fallout of the COVID-19 shutdown from a musculoskeletal standpoint.
\end{abstract}

Keywords: Musculoskeletal discomforts, pain, COVID-19, pandemic

\section{Musculoskeletal discomfort in the wake of COVID-19}

A lack of physical activity has been extensively linked to the development and exacerbation of musculoskeletal discomforts (MSDs) [1, 2]. Fibromyalgia syndrome, a disorder of chronic non-articular musculoskeletal pain and discomfort, is treated with first-line addition of rehabilitation and exercise programs, before proceeding to pharmaceutical intervention [3]. Exercise can be extremely effective for

\footnotetext{
*Address for correspondence: Dr. Ardalan Shariat, Sports Medicine Research Center, Neuroscience Institute, Tehran University of Medical Sciences, Tehran, Iran. Tel.: +982188630227; E-mail: Ardalansh2002@gmail.com.
}

this condition [3]. Despite the obvious benefits of exercise, overuse and improper form while participating in an exercise routine can lead to the onset of MSDs and joint inflammation due to overuse [4].

Quarantine and economic shutdowns due to COVID-19 has led to the creation of various exercise routines by celebrities, athletes, and lay persons, which have been distributed via social media outlets. Individuals will simply utilize a smartphone to generate a video of their favorite exercise done at home. Videos which are then posted to YouTube, Facebook, or Instagram will sometimes receive hundreds of thousands or even millions of views by people around the world who have been confined to the boundaries of their home. There is no regulation of 
these exercise regimens, and while many are harmless, the widespread distribution of these materials coupled with the lack of access to full gymnasium equipment and personal trainers who have been educated on proper and safe execution of exercise, raises several questions in the minds of experts in the field, such as:

- For which age groups are these exercises prepared for?

- If a viewer has a musculoskeletal problem such as low back pain or arthritis, what will the ramifications be for participation in these exercises?

- Are there individuals who may be able to provide proper instructions and guidance for people who wish to participate in these exercise regimens?

- Are there ways to manage the accuracy of exercise movements?

- If physical issues arise from overuse, improper completion of exercise, or widespread distribution of generally harmful physical movements, how will providers respond to increased demand?

The initial shift towards virtual communications began with the introduction of telemedicine [5]. These evaluations can also be done in the field of sports medicine for the best outcome in online exercise prescription (5). Providers should prepare for a 'new normal' phase of life, where a growing number of people will have different types of MSDs. We believe that a sedentary lifestyle during quarantine and practicing exercises obtained online without any confirmed guidelines could impose side effects irrespective of the good intentions of their initial distribution. Low back pain as a major public health problem with lifetime prevalence of $84 \%$ is one of the most important concerns in patients during quarantine [6]. People with different grades of low back pain have varying exercise quantities and type recommendations, and there are no modifications of online prescribed exercises to fit these specifications.

Therefore, we call for the creation of a set of guidelines for the lay person to utilize in the wake of the COVID-19 pandemic. These guidelines could provide exact time and intensity recommendations tailored to the sedentary reality of the COVID19 shutdown, adjusting previous recommendations. Additionally, they could shed light on which activities may be prone to the development of overuse injuries or the onset of low back pain. We hope that by creating a 'new-normal' paradigm for home fitness, practitioners can mitigate MSDs related to the COVID-19 pandemic.
A major barrier to individuals with arthritis, low back pain and other MSDs is a belief that particularly weight bearing exercises will exacerbate joint symptoms. These people need to be reassured by physicians in the sports medicine field that a suitable volume of exercise is not only safe but also generally reduces pain and inflammation in painful joints [7].

Long continuous bouts of aerobic exercise may initially be difficult for those who are deconditioned due to the COVID-19 pandemic and have joint-restricted mobility due to pain. It is appropriate to start with short bouts of 10-15 minutes or even less for two or three times a day rather than inactivity. Improving muscular strength and endurance may reduce pain in inflamed joints. It can be started from isometric exercises counting 10 seconds for 10 times per hour each day in muscles around painful joints. As pain and inflammation is subsided in joints, isometrics can be progressed to isokinetic dynamic strength training of large muscle groups for two-three days a week. Flexibility and neuromotor exercises should be incorporated into the exercise regimen of patients with MSDs.

Hence, we want to reinforce that an exercise prescription should be individualized for patients due to their functional capacity and comorbidities, and that a routine exercise regimen should not be used.

\section{Conflict of interest}

The authors declared no potential conflicts of interest with respect to the research, authorship, and/or publication of this article.

\section{References}

[1] Shariat A, Cardoso JR, Cleland JA, Danaee M, Ansari NN, Kargarfard M, et al. Prevalence rate of neck, shoulder and lower back pain in association with age, body mass index and gender among Malaysian office workers. Work. 2018;60(2).

[2] Shariat A, Cleland JA, Danaee M, Kargarfard M, Sangelaji B, Tamrin SBM. Effects of stretching exercise training and ergonomic modifications on musculoskeletal discomforts of office workers: a randomized controlled trial. Brazilian J Phys Ther. 2018;22(2).

[3] Sosa-Reina MD, Nunez-Nagy S, Gallego-Izquierdo T, PecosMartín D, Monserrat J, Álvarez-Mon M. Effectiveness of therapeutic exercise in fibromyalgia syndrome: a systematic review and meta-analysis of randomized clinical trials. Biomed Res Int. 2017;2017.

[4] Bell DR, Post EG, Biese K, Bay C, McLeod TV. Sport specialization and risk of overuse injuries: a systematic review with meta-analysis. Pediatrics. 2018;142(3):e20180657. 
[5] Ahmed OH, Carmody S, Walker LJ, Ahmad I. The need for speed! 10 ways that WhatsApp and instant messaging can enhance communication (and clinical care) in sport and exercise medicine. BMJ Publishing Group Ltd and British Association of Sport and Exercise Medicine; 2020.
[6] Almoallim H, Alwafi S, Albazli K, Alotaibi M, Bazuhair T. A Simple Approach of Low Back Pain. Int J Clin Med. 2014;2014.

[7] Brosseau L, MacLeay L, Welch V, Tugwell P, Wells GA. Intensity of exercise for the treatment of osteoarthritis. Cochrane database Syst Rev. 2003;(2). 